\title{
Transformation of Meloxicam Containing Nanosuspension into Surfactant-Free Solid Compositions to Increase the Product Stability and Drug Bioavailability for Rapid Analgesia
}

This article was published in the following Dove Press journal:

Drug Design, Development and Therapy

\author{
Csaba Bartos' \\ Rita Ambrus' \\ Gábor Katona' \\ Tamás Sovány (D) \\ Róbert Gáspár (iD) ${ }^{2}$ \\ Árpád Márki (iD) ${ }^{3}$ \\ Eszter Ducza ${ }^{4}$ \\ Anita Ivanov ${ }^{4}$ \\ Ferenc Tömösi (iD ${ }^{5}$ \\ Tamás Janáky (1D ${ }^{5}$ \\ Piroska Szabó-Révész (D) \\ 'Faculty of Pharmacy, Institute of \\ Pharmaceutical Technology and \\ Regulatory Affairs, University of Szeged, \\ Szeged, Hungary; ${ }^{2}$ Faculty of Medicine, \\ Department of Pharmacology and \\ Pharmacotherapy, University of Szeged, \\ Szeged, Hungary; ${ }^{3}$ Faculty of Medicine, \\ Department of Medical Physics and \\ Informatics, University of Szeged, Szeged, \\ Hungary; ${ }^{4}$ Faculty of Pharmacy, \\ Department of Pharmacodynamics and \\ Biopharmacy, University of Szeged, \\ Szeged, Hungary; ${ }^{5}$ Interdisciplinary \\ Excellence Centre, Department of \\ Medical Chemistry, University of Szeged, \\ Szeged, Hungary
}

Purpose: The aim of this work was to study the influence of solidification of meloxicam (Mel) containing nanosuspension (nanoMel) on the physical stability and drug bioavailability of the products. The nanoMel sample had poly(vinyl alcohol) (PVA) as a protective polymer, but no surfactant as a further stabilizing agent because the final aim was to produce surfactant-free solid phase products as well.

Methods: The solidified samples produced by fluidization and lyophilization (fluidMel, lyoMel) were examined for particle size, crystallinity, and in vitro release of Mel compared to similar parameters of nanoMel. The products were subjected to an animal experiment using per oral administration to verify their bioavailability.

Results: Mel containing (1\%) nanoMel sample was produced by wet milling process using an optimized amount of PVA $(0.5 \%)$ which resulted in $130 \mathrm{~nm}$ as mean particle size and a significant reduction in the degree of crystallinity $(13.43 \%)$ of Mel. The fluidization technique using microcrystalline cellulose (MCC) as carrier resulted in a quick conversion and no significant change in the critical product parameters. The process of lyophilization required a longer operation time, which resulted in the amorphization of the crystalline carrier (trehalose) and the recrystallization of $\mathrm{Mel}$ increased its particle size and crystallinity. The fluidMel and lyoMel samples had nearly five-fold higher relative bioavailability than nanoMel application by oral administration. The correlation between in vitro and in vivo studies showed that the fixed Mel nanoparticles on the surface of solid carriers (MCC, trehalose) in both the artificial gastric juice and the stomach of the animals rapidly reached saturation concentration leading to faster dissolution and rapid absorption.

Conclusion: The solidification of the nanosuspension not only increased the stability of the Mel nanoparticles but also allowed the preparation of surfactant-free compositions with excellent bioavailability which may be an important consideration for certain groups of patients to achieve rapid analgesia.

Keywords: solidification, fluidization, lyophilization, surfactant-free product, rapid drug absorption, IVIV correlation

\section{Introduction}

Nanosuspensions can be defined as colloidal dispersions of nanosized drug particles $(<500 \mathrm{~nm})$ that are produced by different nanonization processes and stabilized by various excipients. ${ }^{1}$ Nanonization of drugs with different top-down methods (wetbead milling, high-pressure homogenization and microfluidization) is a proven
Correspondence: Piroska Szabó-Révész Faculty of Pharmacy, Institute of Pharmaceutical Technology and Regulatory Affairs, University of Szeged, Eötvös u 6, Szeged H-6720, Hungary

Tel +36 625455722

Fax +3662545571

Email revesz@pharm.u-szeged.hu 
effective strategy to decrease the particle size by mechanical processes and to enhance the dissolution rate, saturation solubility and bioavailability of poorly water-soluble active ingredients, such as BCS class II (poorly soluble and high permeable) and Class IV (poorly soluble and permeable). ${ }^{2,3}$ Nanosuspensions produced by milling are generally unstable; therefore, stabilizing agents (polymers, surfactants) and its transformation to the solid-state have an important role in the formulations with long-term stability. $^{4,5}$ Water-soluble polymers, such as $2.4-19.6 \%$ of cellulose ethers, ${ }^{6} 30 \%$ of poly(vinyl pyrrolidone), ${ }^{7,8}$ and $50 \%$ of poly(vinyl alcohol), ${ }^{9,10}$ are mainly used in wet milling. The most commonly used surfactants and their amount in relation to the amount of active ingredient are as follows: Cremophor ${ }^{\mathrm{R}}(100 \%),{ }^{11}$ Poloxamer 188 $(60 \%),{ }^{12}$ Poloxamine $\left.908(20 \%)\right)^{13}$ Tyloxapol $(20 \%){ }^{14}$ sodium lauryl sulfate $(0.15 \%),{ }^{15}$ and Polysorbate 80 $(1 \%){ }^{16,17}$ In the absence of stabilizers, the high surface energy of nanosized drug particles can induce aggregation/ agglomeration in the system. ${ }^{18}$ The main functions of stabilizers in nanosuspensions are to wet drug particles during the milling process and to prevent Ostwald's ripening (crystal growth in colloidal suspensions) ${ }^{19}$ and agglomeration in order to yield a physically stable formulation by providing steric or ionic barriers. Different concentrations of stabilizer agents (eg, polymers) can also influence the viscosity and the electro-kinetic property of the particles, according to the DLVO theory, ${ }^{20}$ and thus the stability of the nanosuspension as well. Surfactants help to wet the particles and thus reduce their aggregation tendency. In addition to the advantages of surfactants, they have the biggest disadvantage of increasing the speed/ energy of motion of the milling balls during wet milling, which can lead to the degradation of the active ingredient. When used as an external surfactant to solidify the nanosuspension, its solubility-enhancing effect may be emphasized, thereby increasing the degree of crystallinity of active agent in the solid product and reducing its dissolution rate. ${ }^{21}$ Conventional formulations contain these excipients in common, but the new tendency is to ignore the surfactants and look for other options to stabilize the nanoparticles in the products and achieve the desired biological effect. ${ }^{22-24}$

Crystalline state is one of the most important parameters affecting drug stability, dissolution extent, and efficacy. The high energy wet milling techniques tend to create a partially amorphous active agent. The high energy amorphous particles are unstable, especially in the presence of crystalline particles, and inclined to convert to low energy crystalline state over time. The saturation solubility between amorphous and crystalline nanoparticles is different; therefore, the diffusion process will be similar to Oswald's ripening, leading to a rapid conversion of amorphous nanoparticles to crystalline state. ${ }^{25}$

Of course, the nanosuspensions can be applied as final liquid dosage forms using further different excipients (viscosity enhancer, flavoring, preservative agents, etc.); however, their stabilization is a major challenge. ${ }^{26}$ It is well known that, despite the stabilization, nanosuspensions have a short expiration time, and there are patients who do not prefer this form or the presence of a surfactant. One way to overcome the instability and surfactant problem is to design solid nanosuspension produced by spray drying, spray freeze drying and freeze drying (lyophilization). It is well known that dry nanosuspensions can cause difficulty in hydration and redispersibility. ${ }^{24,27}$ Other processes for transforming a nanosuspension into solid-state forms (tablets, capsules) are very different: deposition as coatings, incorporation in granules and pellets and the $3 \mathrm{D}$ printing technologies. ${ }^{28}$

Spray drying and lyophilization (freeze drying) are the commonly used techniques for the solidification of nanosuspension because of their easy application and industrial acceptability. ${ }^{29}$ The powders produced by these processes often suffer from poor flowability and high hygroscopicity; therefore, other technologies are applied to transform nanosuspensions into oral dosage forms as tablets and capsules. $^{30}$

Layering of nanosuspension onto the surface of granules, pellets, sugar beads, etc., using a fluidization technique is used as an alternative method for solidification of nanosuspension. ${ }^{30-32}$ The advantages of this process that may use various additives in order to achieve the desired purpose are as follows: (i) different polymers stabilize the nanosuspension and act as coating materials resulted in fast dissolution rate; (ii) surfactants prevent the aggregation of nanoparticle and can modify the drug release. ${ }^{15}$ In any case, the fluidization technique also provides an opportunity to stabilize the broken structure of the ground crystals in the nanosuspension by using crystallization inhibitors. $^{33}$

Mel, a member of the oxicam family of NSAIDs (nonsteroidal anti-inflammatory drugs), as a moderately selective cyclooxygenase (COX-2) inhibitor can have a role in acute pain therapy, but a basic requirement is a rapid absorption through the gastric mucosa. Mel has a weak 
acidic character with $\mathrm{pKa}$ of 3.43 ; therefore, its solubility in gastric juice $(\mathrm{pH}=1.2)$ is very poor but its logPapp is $2.43(\mathrm{pH}=2.0)$, which predestines the fast absorption from the stomach. ${ }^{34-36}$ Since the solubility of Mel is very poor in gastric juice, the preparation of a nanosuspension with a fast dissolution rate may be a solution.

In our previous work, we developed a wet milling procedure for the nanonization of Mel, which was reported in the DDDT in $2018 .{ }^{10}$ We optimized the critical process parameters by factorial design (ratio of predispersion and pearls, milling time and rotation speed of the steel jar) and investigated the PVA amount on the particle size distribution and crystallinity of the Mel. The optimized process parameters and PVA amount have allowed the use of no surfactant during milling to prevent aggregation. We had also the surfactant-free nanosuspension as intermediate product showed a stable system for critical product parameters with 2 weeks of holding time.

The main purpose of the work was to produce a surfactant-free product by solidifying of Mel-containing nanosuspension. Critical product parameters were considered to be the particle size distribution of the drug $(\mathrm{d}(0.9)$ $<500 \mathrm{~nm}$ ), stabilization of the degree of crystallinity altered during milling, and enhancement of the bioavailability of the solid product with fast absorption from the stomach for rapid analgesia. The transformation of the nanosuspension was done by fluidization and lyophilization.

\section{Materials and Methods Materials}

Mel (rawMel) was obtained from EGIS Ltd. (Budapest, Hungary). PVA-Mowiol ${ }^{\mathrm{R}}$ 4-98 (Mw 27,000) (Sigma Aldrich Co. LLC, St. Louis MO, USA) was used as a stabilizing agent. Zirconium oxide $\left(\mathrm{ZrO}_{2}\right)$ beds with a diameter of $0.30 \mathrm{~mm}$ were obtained from Netsch (Netsch GmbH, Selb, Germany). Microcrystalline cellulose (MCC) (Avicel $^{\mathrm{R}}$ PH 101, FMC Biopolymer, Philadelphia USA) was used as a carrier material for the fluidized product. D-(+)trehalose dihydrate as a cake-forming agent was purchased from Karl Roth GmbH + Co. KG. (Karlsruhe, Germany).

\section{Methods}

Preparation of Nanosuspension (nanoMel)

For the production of the Mel nanosuspension, a planetary ball mill was combined with pearl milling technology. PVA was used as a stabilizing additive, $1.0 \mathrm{~g}$ of PVA was dissolved in $17.0 \mathrm{~g}$ of distilled water as a dispersant medium, in which
$2.0 \mathrm{~g}$ of Mel was suspended. The milling was executed using a Retsch PM 100 planetary ball mill (Retsch GmbH, Haan, Germany) at $437 \mathrm{rpm}$ rotation speed for $43 \mathrm{~min}$ in addition with $20.0 \mathrm{~g}$ of $0.3 \mathrm{~mm} \mathrm{ZrO}_{2}$ beads as a milling medium. After the milling process, to eliminate the grinding medium from the sample, a $0.150-\mathrm{mm}$ sieve was used. The nanosuspension was removed from the beads by cleaning with distilled water, while the milled sample was ten-fold diluted. The yield of the milling process was $94.93 \%$. The final concentration of the components can be seen in Table 1.

\section{Transformation of Nanosuspension into Solid-State Form}

In the preformulation study, surfactant-free and external surfactant-containing (Polysorbate 80) samples were produced by fluidization to study the critical parameters of the products. It was found that the external surfactant used to solidify the nanosuspension (nanoMel) increased the degree of crystallinity and decreased the dissolution rate of Mel. This change is related to the solubility-enhancing effect of the surfactant. It was concluded that, in the absence of surfactant, the critical product parameters can be fulfilled by optimization of process parameters of fluidization and lyophilization.

\section{Fluidization (fluidMel)}

MCC as the carrier material was used in a Strea-1 (Niro Aeromatic, Bubendorf, Switzerland) fluid-bed chamber. A batch size of $100.0 \mathrm{~g}$ was used. The powder was inserted and fluidized in the preheated chamber for a period of $10 \mathrm{~min}$ and at a constant air velocity of $2.5 \mathrm{~m} / \mathrm{s}$. NanoMel as liquid dispersion was transported by a peristaltic pump (Roto Consulta, Ebikon/Luzern, Schweiz), the applied pump speed was $9 \mathrm{rpm}$. One batch of $200.0 \mathrm{~g}$ of nanoMel dispersion was atomized onto the surface of the material. The process took $50 \mathrm{mins}$, the inlet temperature was $55^{\circ} \mathrm{C}$ and the outlet temperature was $38^{\circ}$ C. The final concentration of the components in percentage is shown in Table 1 . The yield of the process was

Table I Composition of the Investigated Samples

\begin{tabular}{|l|l|l|l|l|l|}
\hline Sample & $\begin{array}{l}\text { Mel } \\
(\%)\end{array}$ & $\begin{array}{l}\text { PVA } \\
(\%)\end{array}$ & $\begin{array}{l}\text { Water } \\
(\%)\end{array}$ & $\begin{array}{l}\text { MCC } \\
(\%)\end{array}$ & $\begin{array}{l}\text { Trehalose } \\
(\%)\end{array}$ \\
\hline nanoMel & 1.00 & 0.50 & 98.50 & - & - \\
fluidMel $^{\mathrm{a}}$ & 1.94 & 0.97 & - & 97.09 & - \\
lyoMel $^{\mathrm{b}}$ & 15.38 & 7.70 & - & - & 76.92 \\
\hline
\end{tabular}

Notes: ${ }^{\mathrm{a}, \mathrm{b}}$ Based on dry material.

Abbreviations: Mel, meloxicam; PVA, poly(vinyl alcohol); MCC, microcrystalline cellulose. 
calculated based on the proportion of the mass of the components before and after the operation.

\section{Lyophilization (lyoMel)}

Freeze-drying was performed in Scanvac CoolSafe 100-9 Pro type equipment (LaboGene ApS, Lynge, Denmark) equipped with a 3 -shelf sample holder unit, recessed into the drying chamber. In each cuvette $750 \mathrm{mg}$ of diluted milled suspension was filled ( $7.5 \mathrm{mg}$ Mel content in every cuvette, the unit dose of Mel). As a cake-forming additive, $37.5 \mathrm{mg}$ of $(5.0 \%)$ trehalose was dissolved in the nanosuspensions. The components of the final product can be seen in Table 1. The process was controlled by a computer program (Scanlaf CTS16a02), the temperature and pressure values were recorded continuously. The whole process took $71 \mathrm{hrs}$ and 52 mins. The initial temperature was $25^{\circ} \mathrm{C}$. During the freezing period, after $18 \mathrm{hrs}$ and $34 \mathrm{mins}$, the sample temperature was decreased to $-40^{\circ} \mathrm{C}$. The subsequent drying process was conducted at 0.013 mbar air pressure for $50 \mathrm{hrs}$ and $50 \mathrm{mins}$, the temperature increased from $-40^{\circ} \mathrm{C}$ to $-7^{\circ} \mathrm{C}$. Finally, the secondary drying session took $2 \mathrm{hrs}$ and 28 mins. The final temperature of the dried products was $30^{\circ} \mathrm{C}$.

\section{Characterization of Nanosuspension and the Solid-State Forms}

\section{Particle Size Measurements}

The investigations on the particle size of rawMel el and nanoMel via laser diffraction were executed (Malvern Mastersizer S 2000, Malvern Instruments Ltd, Worcestershire, UK) with the following parameters: 300RF lens; small volume dispersion unit $(1,000 \mathrm{rpm})$; refractive index for Mel: 1.596; and refractive index for dispersion medium: 1.330. During the measurements, distilled water was used as a dispersant, and obscuration was in the range of $11-16 \%$ for all measurements. In both cases, the particle size distributions were qualified by $\mathrm{d}(0.1), \mathrm{d}(0.5)$, and $\mathrm{d}(0.9)(\mathrm{d}$ (0.5) is the particle diameter below which $50 \%$ of the sample volume exists).

In the case of the solid-state products (fluidMel and lyoMel), the particle size of Mel was determined by using Scanning Electron Microscopy (SEM) images (Hitachi S4700, Hitachi Scientific Ltd., Tokyo, Japan). The size of the particles was calculated by ImageJ software for Windows (Phase GmbH, Lübeck, Germany). ${ }^{37}$ By specifying the unit length, which is shown in each image (depending on the magnification this is a different value), the actual particle size can easily be determined by drawing the diameter of the captured particles. A diameter of a hundred captured particles was determined in the case of two solid-state samples.

For nanoMel and lyoMel samples, the Z-average particle size and the polydispersity index (PDI) of Mel were measured using a Malvern Zeta Nano ZS (Malvern Instruments Ltd). In case of the particle size determination, Malvern DTS 1070 folded capillary cell was used. The samples were further diluted with water (25-fold) for the measurements.

\section{Morphology of the Samples (SEM)}

For SEM investigations, nanoMel samples were dried in a vacuum dryer (Binder $\mathrm{GmbH}$, Tuttlingen, Germany) at $40^{\circ} \mathrm{C}$ in order to obtain solid products, and fluidMel and lyoMel were visualized as well. The samples were sputtercoated with gold-palladium under an argon atmosphere, using a gold sputter module in a high-vacuum evaporator, and the samples were examined at $10 \mathrm{kV}$ and $10 \mathrm{~mA}$. The air pressure was 1.3-13 MPa.

\section{Differential Scanning Calorimetry (DSC)}

To investigate the occurring physico-chemical changes and to predict the crystallinity of the solid-state products, DSC measurements were carried out with a Mettler Toledo DSC 821e thermal analysis system with the STARe thermal analysis software V9.0 (Mettler Inc. Schwerzenbach, Switzerland). Approximately $2-5 \mathrm{mg}$ of the physical mixtures (PM) and the product samples were examined in the temperature range of $25-300^{\circ} \mathrm{C}$. The heating rate was $20^{\circ} \mathrm{C} / \mathrm{min}$ in the presence of argon as a carrier gas with a flow rate of $10 \mathrm{~L} / \mathrm{h}$. The calculations of Mel crystallinity (Cryst \%) were performed using the area under the curve (AUC) of the melting enthalpy of the products (AUCMel) and the physical mixtures (AUCMelPM) ${ }^{38}$ The values were compared using the following formula:

$$
\text { Cryst } \%=\frac{\text { AUCMel }}{\text { AUCMelPM }} \times 100
$$

\section{Stability Test}

The products (fluidMel and lyoMel) were stored in a wellclosed container, at room temperature $\left(23 \pm 2^{\circ} \mathrm{C}, 45 \pm 5 \%\right.$ $\mathrm{RH})$ for 6 months. The crystallinity of Mel was investigated compared to freshly measured products.

\section{Drug Content Determination}

The Mel content of the samples was controlled in the following way. The unit dose of the products with 
$0.75 \mathrm{mg}$ of theoretical Mel was dissolved in $100 \mathrm{~mL}$ of phosphate buffer $\mathrm{pH} 7.4 \pm 0.1$. The sample was stirred with a magnetic stirrer at $25^{\circ} \mathrm{C}$ for $24 \mathrm{hrs}$ and then filtered $(0.1$ $\mu \mathrm{m}$, FilterBio PES Syringe Filter) (Labex Ltd., Budapest, Hungary), and the concentration of the dissolved Mel was analyzed spectrophotometrically (Unicam UV/VIS) (Thermo Fisher Scientific Inc., Waltham, MA, USA) at $364 \mathrm{~nm}$ wavelength. The investigations were repeated three times.

\section{Solubility Testing of MEL in the Samples}

The solubility of Mel in the samples (nanoMel, fluidMel and lyoMel) was determined. The dispersions were stirred with a magnetic stirrer at $25^{\circ} \mathrm{C}$ for $24 \mathrm{hrs}$ and then filtered (0.1 $\mu \mathrm{m}$, FilterBio PES Syringe Filter) (Labex Ltd., Budapest, Hungary), and the dissolved drug content was analyzed spectrophotometrically (Unicam UV/VIS) (Thermo Fisher Scientific Inc., Waltham, MA, USA) at $364 \mathrm{~nm}$ wavelength $(\mathrm{n}=3)$.

\section{In vitro Dissolution Test}

To determine the dissolution extent of Mel from different products, the paddle method (USP dissolution apparatus, type II Pharma Test, Hainburg, Germany) was used. The medium was $900 \mathrm{~mL}$ of artificial gastric fluid at $\mathrm{pH} 1.2 \pm$ 0.1 . The paddle was rotated at $100 \mathrm{rpm}$ and sampling was performed up to $120 \mathrm{~min}$. The Mel content of the samples was determined with a spectrophotometer (ATI-UNICAM UV/ VIS Spectrophotometer, Cambridge, UK) at $362 \mathrm{~nm}$. The number of parallels was three.

\section{Statistical Analyses}

Data from the above methods were expressed as means $\pm \mathrm{SD}$, and groups were compared by using Student's $t$-test. Differences were considered statistically significant when $\mathrm{p}<0.05$.

\section{In vivo Studies}

All experiments involving animal subjects were carried out with the approval of the National Scientific Ethical Committee on Animal Experimentation (permission number: IV/1247/ 2017). The animals were treated in accordance with the European Communities Council Directives (2010/63/EU) and the Hungarian Act for the Protection of Animals in Research (Article 32 of Act XXVIII). Each sample contained $60 \mu \mathrm{g} / \mathrm{mL}$ of Mel and $30 \mu \mathrm{g} / \mathrm{mL}$ of PVA in distilled water. For per os delivery, the different formulations were individually diluted and were given at a single dose of $300 \mu \mathrm{g} / \mathrm{kg}$ of Mel to male Sprague-Dawley rats ( 8 weeks old, 240-260 g, $\mathrm{n}=6$ ) in a volume of $0.5 \mathrm{~mL}$ by gastric gavages. All animals fasted 16 hrs before the per os administration of drugs. In order to facilitate the absorption, the solid-state forms were redispersed in water immediately before administration. In a comparison study for intravenous administration, animals were treated with a $300 \mu \mathrm{g} / \mathrm{kg}$ bolus of Mel via the tail vein.

Intravenous (IV) injection was prepared by the dilution of passable injection with a concentration of $15 \mathrm{mg} / 1.5 \mathrm{~mL}$ (Meloxicam-Zentiva, Prague, Czech Republic) to reach the final concentration $(0.15 \mathrm{mg} / \mathrm{mL})$. The ingredients of the injection were meglumine, poloxamer 188, glycine, sodium hydroxide (for $\mathrm{pH}$ adjustment), sodium chloride, glycopherol, and water for injection. Blood samples were collected from the tail vein before and at 15, 30, 60, 75, 90, 120 and 180-mins post-dosing. Plasma samples were collected into EDTA containing polyethylene tubes, centrifuged at $1,500 \mathrm{~g}$ for $10 \mathrm{~min}$ at $5^{\circ} \mathrm{C}$. Separated plasma samples were stored at $-80^{\circ} \mathrm{C}$ until extraction and analysis.

\section{Determination of Mel from Rat Plasma}

Preparation of plasma samples, calibration standards and quality control samples

To $90 \mu \mathrm{L}$ of plasma sample, $10 \mu \mathrm{L}$ of $0.1 \%$ aqueous formic acid and $300 \mu \mathrm{L}$ of acetonitrile containing piroxicam (internal standard at $12.5 \mathrm{ng} / \mathrm{mL}$ concentration) were added and the mixture was vortex-mixed for $60 \mathrm{~s}$. The mixture was allowed to rest for 30 mins at $-20^{\circ} \mathrm{C}$ to support protein precipitation. The supernatant was obtained by the centrifugation of the mixture for $10 \mathrm{~min}$ at $10,000 \mathrm{~g}$ at $4^{\circ} \mathrm{C}$ and $20 \mu \mathrm{L}$ was diluted with $380 \mu \mathrm{L}$ of $0.1 \%$ aqueous formic acid. Finally, $5 \mu \mathrm{L}$ was injected into the LC-MS/MS system for analysis.

Rat plasma calibration standards of meloxicam were prepared by spiking the working standard solutions (1-1000 $\mathrm{ng} / \mathrm{mL}$ ) into a pool of drug-free rat plasma and the procedure described above was followed. Calibration standards consisted of $90 \mu \mathrm{L}$ of pooled drug-free plasma, $10 \mu \mathrm{L}$ of meloxicam standard solution (in $0.1 \%$ aqueous formic acid) and $300 \mu \mathrm{L}$ of acetonitrile containing piroxicam (internal standard at $12.5 \mathrm{ng} / \mathrm{mL}$ concentration). Solutions containing $6.25 \mathrm{ng} / \mathrm{mL}$ and $25 \mathrm{ng} / \mathrm{mL}$ of meloxicam were used as QC samples. $20 \mu \mathrm{L}$ of supernatant was taken out from both of the calibration standards and the QC samples, diluted with $380 \mu \mathrm{L}$ of $0.1 \%$ aqueous formic acid, and $5 \mu \mathrm{L}$ was analyzed by LC-MS/MS. 


\section{LC-MS/MS Analysis of Meloxicam}

The quantitative analysis of meloxicam was performed by mass spectrometry after the chromatographic separation of analytes. An Agilent Liquid Chromatography System series 1100 (Micro Vacuum Degasser, Capillary Pump, $\mu$-WPS autosampler) (Agilent Technologies, Waldbronn, Germany) was connected to a Q ExactiveTM Plus Orbitrap mass spectrometer (Thermo Fisher Scientific, San Jose, US) equipped with a heated ESI ion source. Gradient chromatographic separation was performed at room temperature on a Luna ${ }^{\mathrm{R}} 5 \mu \mathrm{m}$ C8(2) Mercury column $(20 \mathrm{~mm} \times 2.0 \mathrm{~mm})$ protected by a $\mathrm{C} 8$ guard column $(2 \times 2 \mathrm{~mm})$ (Phenomenex, Torrance, USA) using ammonium formate $(15 \mathrm{mM}, \mathrm{pH}=3)$ as Solvent A and acetonitrile as Solvent B (Table 2). The calibration curve was shown to be linear over the concentration range of $1-1000 \mathrm{ng} / \mathrm{mL}$

The mass spectrometer was used in positive mode with the following parameters of H-ESI source: ion spray voltage at $3.5 \mathrm{kV}$, capillary temperature at $253^{\circ} \mathrm{C}$ and aux gas heater temperature at $406^{\circ} \mathrm{C}$, sheath gas flow rate at $46 \mathrm{l} / \mathrm{h}$, aux gas flow rate at $11 \mathrm{l} / \mathrm{h}$, sweep gas flow rate at $2 \mathrm{l} / \mathrm{h}$ and S-lens RF level at 50.0 (source auto-defaults). Multiplereaction-monitoring (MRM) mode was used for quantification by monitoring the transitions: $\mathrm{m} / \mathrm{z} \quad 352 \rightarrow 115$ and $352 \rightarrow 141$ for meloxicam (collision energy $24 \mathrm{~V}$ ) and $\mathrm{m} / \mathrm{z}$ $332 \rightarrow 95$ and $332 \rightarrow 121$ for piroxicam (collision energy 29 V). A divert valve placed after the analytical column was programmed to switch flow onto MS only when analytes of interest elute from the column (plasma samples: 0.7-2.0 mins) to prevent the excessive contamination of the ion source and ion optics.

Data acquisition and processing were carried out using Xcalibur and Quan Browser (version 4.0.27.19) software (Thermo Fisher Scientific, San Jose, US).

Table 2 The Gradient Elution Program Applied for Analysis

\begin{tabular}{|l|l|l|}
\hline $\mathbf{t}(\mathbf{m i n})$ & B (\%) & Flow Rate $(\boldsymbol{\mu L} / \mathbf{m i n})$ \\
\hline 0 & 40 & 250 \\
0.5 & 40 & 250 \\
2 & 70 & 250 \\
2.1 & 90 & 600 \\
2.5 & 90 & 600 \\
2.6 & 40 & 600 \\
4.0 & 40 & 600 \\
4.1 & 40 & 250 \\
4.5 & 40 & 250 \\
\hline
\end{tabular}

Abbreviation: B, solvent (acetonitrile).
The area under the curve (AUC) of the time (min)concentration $(\mathrm{ng} / \mathrm{mL})$ curves of each animal and the statistical analysis were performed with Prism 5.0 software (GraphPad Software Inc., La Jolla, CA, USA). All data presented are means $\pm \mathrm{SD}$. The unpaired $t$-test was used to determine statistical significance. Changes were considered statistically significant at $p<0.05$. The ratio of AUC value, after the per oral application of the transformed samples (AUCfluidMel, AUClyoMel) in comparison with the AUC of the peroral application of nanoMel (AUCnanoMel) as relative bioavailability (rel.BA) was determined according to the formula below: ${ }^{39}$

rel. BA for plasma $(\%)=\frac{\text { AUCtransformed sample }}{\text { AUCnanoMel }} \times 100$

\section{In vitro-in vivo Correlation Calculation}

In vitro-in vivo correlation (IVIVC) is a biopharmaceutical tool for the investigation of the mutual relationship of the dissolution characteristics of the in vitro and in vivo absorption studies. ${ }^{40}$ In our case, the Pearson's correlation coefficient of the AUC values of the in vitro and in vivo results was calculated by Microsoft Excel (Microsoft Corporation, Redmond, Washington, US) and Statistica for Windows (StatSoft GmbH, Hamburg, Germany). The three prepared samples were correlated with each other in groups of in vitro and in vivo. To determine statistical significance, the unpaired $t$-test was used.

\section{Results and Discussion}

\section{Particle Size Measurements}

Different techniques have been used to determine the particle size of Mel for reasons of accuracy and comparability. The particle size distribution of the rawMel and nanoMel samples was investigated via laser diffraction. A combined wet milling process resulted in a 200 -fold particle size reduction in the case of nanoMel $(\mathrm{d}(0.50), 130 \pm 5 \mathrm{~nm})$ compared to the raw drug particle size $(\mathrm{d}(0.50), 34.26$ $\pm 4.86 \mu \mathrm{m})$. The nanoMel product showed a monodisperse distribution $(\mathrm{d}(0.10), 67 \pm 1 \mathrm{~nm} ; \mathrm{d}(0.50), 130 \pm 5 \mathrm{~nm} ; \mathrm{d}(0.90)$, $371 \pm 12 \mathrm{~nm})$.

For fluidMel sample, Mel particles adhered to the carrier surface (MCC) was analyzed by the ImageJ technique and the particle size of Mel in the nanoMel and lyoMel samples were compared with dynamic light scattering technique (Malvern nanoZS), too. The results demonstrate that the $\mathrm{d}(0.50)$ value of the Mel nanoparticles measured on the surface of solid phase product 
(fluidMel) does not show a significant difference regarding the $d(0.50)$ value of the nanoMel (Figure 1A). In the case of lyoMel, compared to the Z-average of Mel in nanoMel, a significant difference can already be detected which was caused by the recrystallization of the Mel (Figure 1B). Both samples have the same polydispersity index (nanoMel: 0.273 and lyoMel: 0.287 ) which also confirms the monodispersity of nanoMel and shows the excellent redispersibility of lyoMel. The 6-month storage did not cause any further changes in the mean particle size of the products (Figure $1 \mathrm{~A}$ and $\mathrm{B}$ ).

\section{SEM Measurements}

Figure $2 \mathrm{~A}$ and $\mathrm{B}$ clearly show the particle size difference between the rawMel and nanoMel and the change in the particle habit. The latter particles have smooth surfaces with rounded edges and corners. High mechanical impact results in the fracture and abrasion of the crystals.

During the fluidization process, the nanoparticles are uniformly adhered to the surface of MCC (Figure 2C1). Their habit is the same as that of the nanoparticles in the nanoMel (Figure 2C2). There is no sign indicating the aggregation of the nanoparticles on the surface of MCC. The adhesion of the Mel particles to the carrier surface is also supported by the effect of the PVA adhesive property and the rapid evaporation of water.

The SEM image of the lyoMel sample (Figure 2D1) shows large, consistent formulas at low magnification. The texture at higher magnification contains smaller, larger pores resulting in a big surface with honeycombed structure, where the surface area is determined by the size of the ice crystals. ${ }^{41}$ The SEM picture does not show any trehalose-like crystals in the structure (Figure 2D2).

\section{DSC Measurements, Crystallinity Determination}

The DSC curves of the components and the products are shown in Figure 3. The rawMel has a relatively high melting point at $268^{\circ} \mathrm{C}$, PVA as semi-crystalline material has two endothermic peak at $169^{\circ} \mathrm{C}$ and at $222^{\circ} \mathrm{C}$. MCC shows any characteristic peak; in contrast, the trehalose is a crystalline material (Figure 3A).

The thermograms of the products are shown in Figure $3 \mathrm{~B}$. It is clear that the melting point and the enthalpy of Mel in the case of the nanoMel decreased due to the partial amorphization.
The curve of the fluidMel shows the peak of MCC and the decreased melting point of Mel (Figure 3B). The first obvious and big endothermic peak of the curve appearing from $30^{\circ} \mathrm{C}$ to $150^{\circ} \mathrm{C}$ is mainly related to the absorbed moisture evaporation. $^{42}$ The second endothermic peak is connected to the melting point of $\mathrm{Mel}\left(264^{\circ} \mathrm{C}\right)$ and the enthalpy was decreased due to the large amount of MCC, which covered the characteristic peak of PVA as well, compared to nanoMel.

The curve of lyoMel represents the peak of PVA $\left(197^{\circ} \mathrm{C}\right)$ and the melting temperature of $\mathrm{Mel}\left(251^{\circ} \mathrm{C}\right)$ (Figure $\left.3 \mathrm{~B}\right)$. According to the literature ${ }^{43}$ and our measurements, during the process, the total amount of trehalose transformed into an amorphous form and the lyophilized trehalose maintains its amorphous form. The big endothermic peak of the curve appearing from $30^{\circ} \mathrm{C}$ to $150^{\circ} \mathrm{C}$ is connected to the absorbed water evaporation as well.

The crystallinity of Mel in nanoMel and the transformed solid-state products were calculated by the enthalpy changes of the drug occurring during the DSC measurement (Table 3). Each sample was compared to its own physical mixture. According to the crystallinity of the nanoMel (13.43\%) sample, the crystallinity of the fluidMel sample did not change (12.98\%), for the lyoMel sample partial recrystallization $(40.11 \%)$ occurred.

After 6 months of storage $\left(23 \pm 2^{\circ} \mathrm{C}, 45 \pm 5 \% \mathrm{RH}\right)$, the degree of crystallinity of solidified samples (fluidMel and lyoMel) was determined again. The results did not show a significant change $(\mathrm{p}>0.05)$ compared to the non-stored, fresh samples (Table 3 ). There was no sign for the recrystallization of trehalose.

\section{Drug Content Determination}

The theoretical drug content was $7.50 \mathrm{mg}$ as a single dose/ oral. For the nanoMel sample, this amount was $7.12 \mathrm{mg}$ and fluidMel showed $6.83 \mathrm{mg}$ of Mel. The latter can be related to the yield of the fluidization technique (95.93\%). During the lyophilization process, the Mel content of the sample (lyoMel) was $7.12 \mathrm{mg}$.

\section{Solubility Testing of MEL in the Samples}

The solubility of nanoMel increased significantly $(9.4 \pm 0.5$ $\mu \mathrm{g} / \mathrm{mL})$ in comparison with the rawMel $(6.5 \pm 0.2 \mu \mathrm{g} / \mathrm{mL})$. The reduced particle size enhanced the wettability of the hydrophobic particle when using PVA, therefore increased the thermodynamic solubility of Mel. The fluidization process did not affect the solubility of $\mathrm{Mel}(9.6 \pm 0.4 \mu \mathrm{g} / \mathrm{mL})$. In the case of lyoMel, solubility was increased $(11.2 \pm 0.5 \mu \mathrm{g} / \mathrm{mL})$ because of the presence of trehalose. 

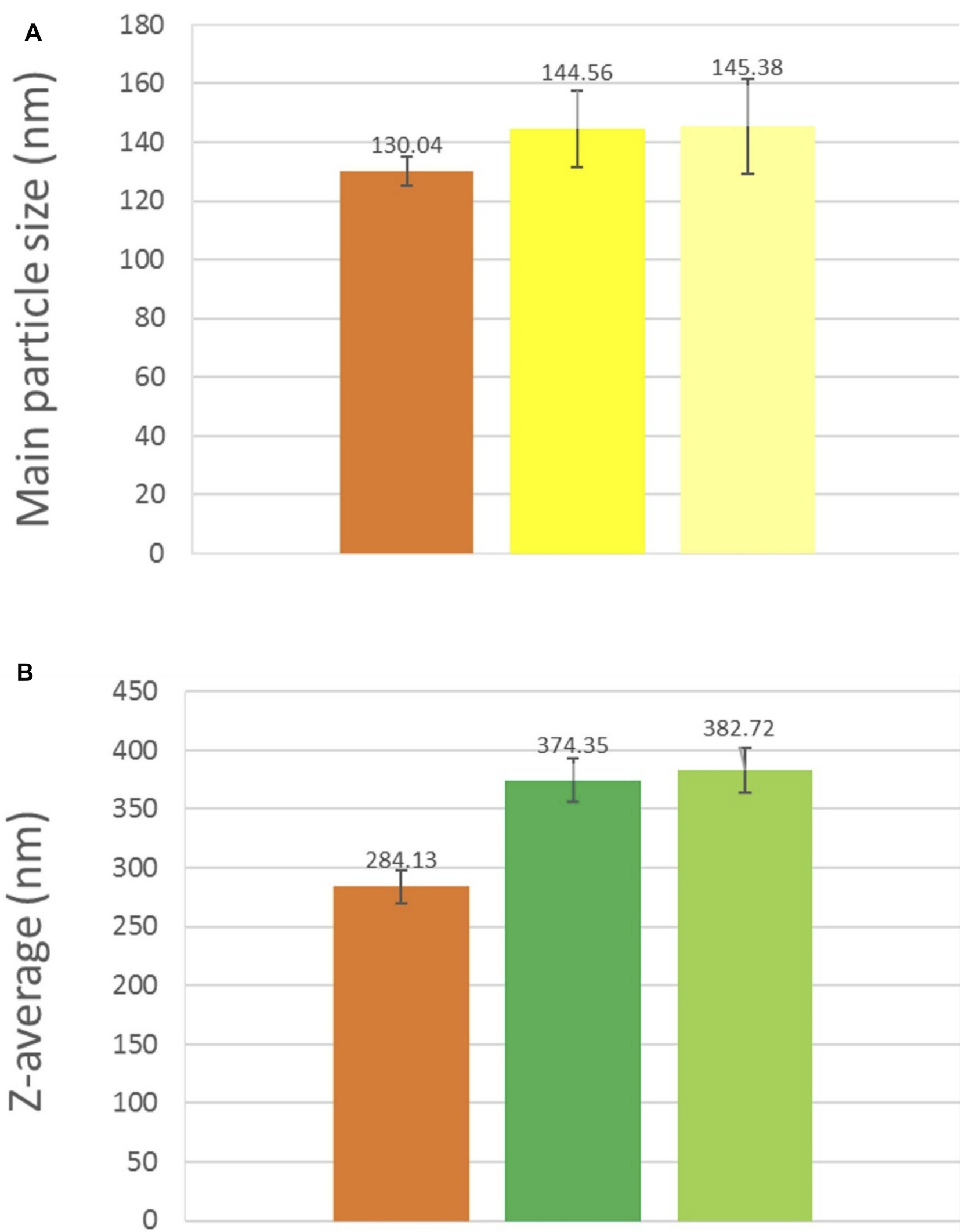

Figure I (From left to the right). Main particle size of nanoMel (measured by laser diffraction), fluidMel fresh and fluidMel stored (6 months) (measured by SEM images) (A) and Z-average of nanoMel, lyoMel fresh, and lyoMel stored (6 months) (measured by Zeta nano ZS) (B).

Abbreviations: Mel, meloxicam; SEM, scanning electron microscopy. 

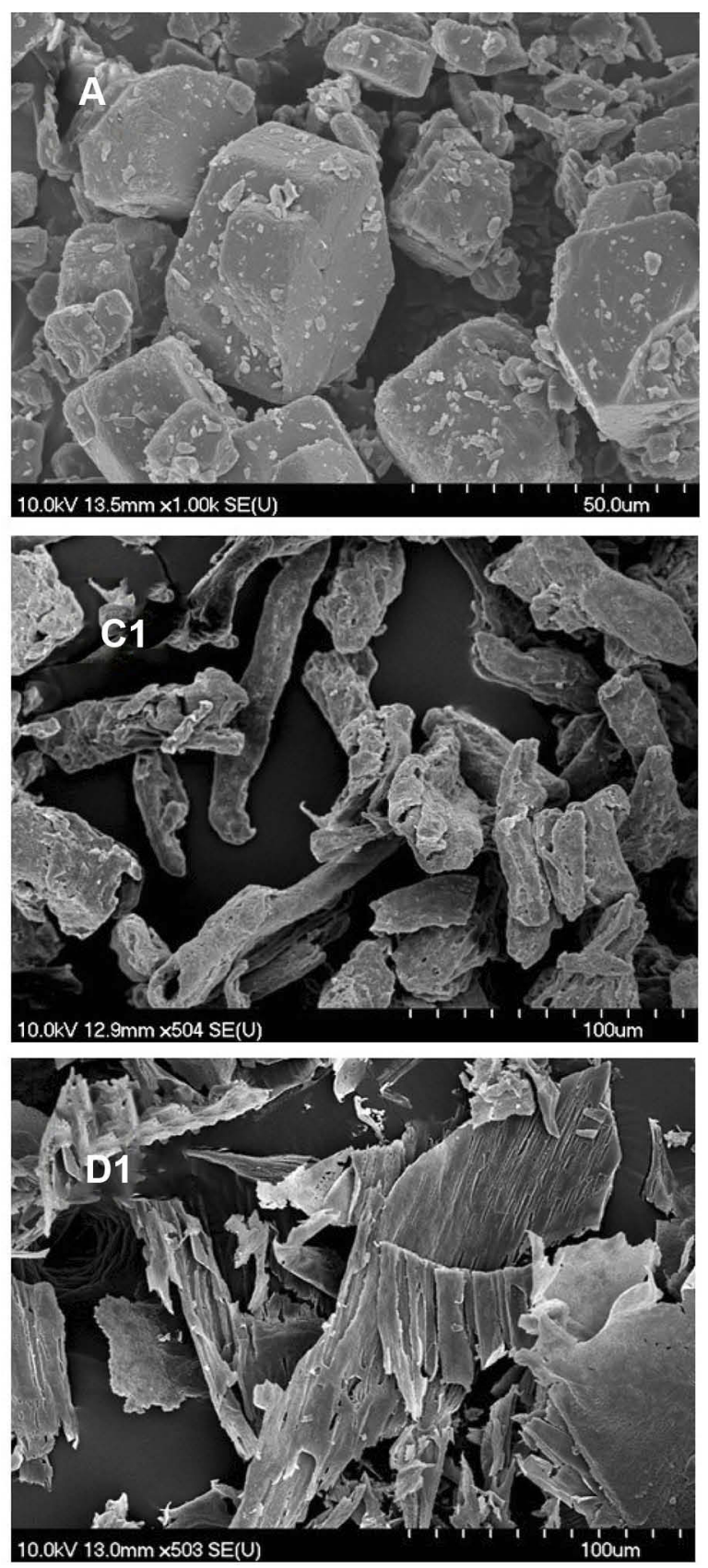
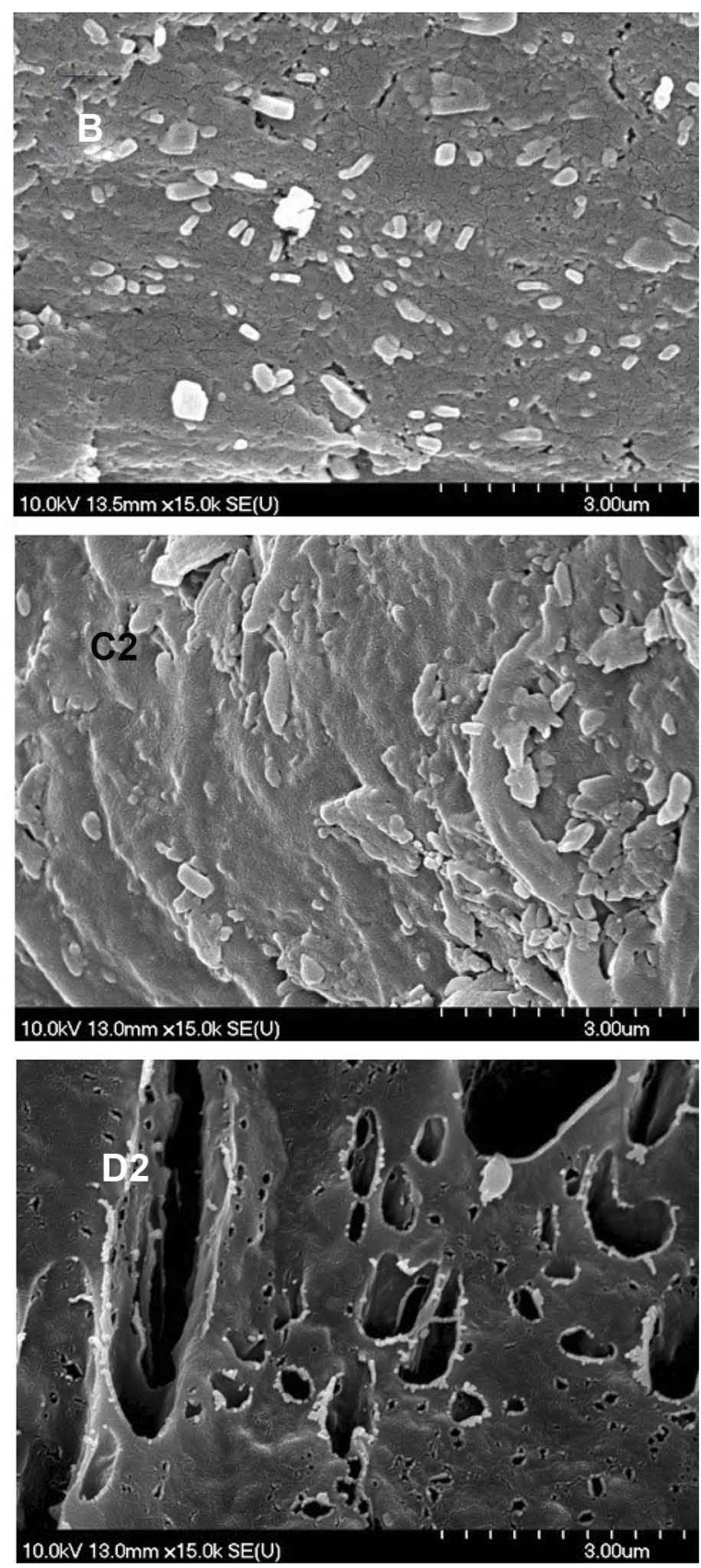

Figure 2 SEM image of rawMel (A), nanoMel (B), fluidMel (CI and C2) and lyoMel (DI and D2).

Abbreviations: Mel, meloxicam; SEM, scanning electron microscopy.

\section{In vitro Dissolution Studies}

The in vitro dissolution extent of the samples was investigated in gastric juice $(\mathrm{pH}=1.2)$. Mel has a week acidic character; therefore, its solubility in this medium is very poor $\left(1.6 \pm 0.2 \mu \mathrm{g} / \mathrm{mL}\right.$, at $\left.37^{\circ} \mathrm{C}\right)$. Figure 4 clearly demonstrates that the particle size reduction of Mel in the nanosuspension (nanoMel) influenced the dissolution rate of Mel but resulted in only $40 \%$ of drug release in 5 mins, and then the curve took a stagnant profile. The initial rapid drug release can be associated with the nanoscale Mel and its amorphous structure. The 2-hrs test did not result in any more favorable results. Although the distribution of the nanoparticles of Mel in the nanosuspension is suitable, a large volume of acidic medium $(900 \mathrm{~mL}$ ) may increase the aggregation of the nanoparticles. In this case, the protective effect of the polymer (PVA) is unsatisfactory.

For fluidMel and lyoMel samples, a rapid initial phase is observed (about $60 \%$ of the drug is dissolved in 15 

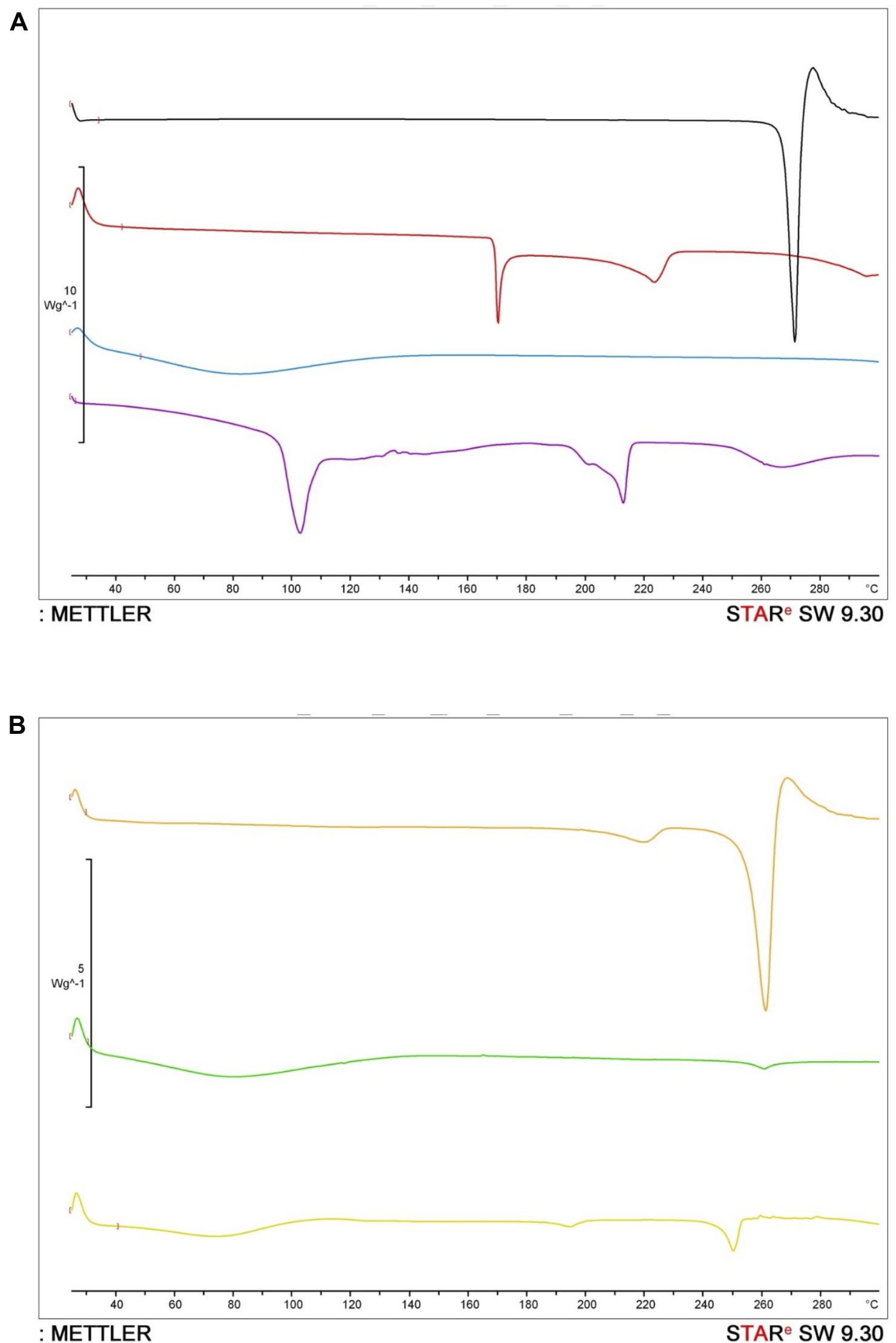

Figure 3 DSC curves of rawMel (black), PVA (red), MCC (blue) and trehalose (purple) (A), and nanoMel (orange) fluidMel (green) and lyoMel (yellow) (B). Abbreviations: DSC, differential scanning calorimetry; Mel, meloxicam; PVA, poly(vinyl alcohol). 
Table 3 Enthalpy and Calculated Crystallinity Values of the Characteristic Peak of Mel in the Samples

\begin{tabular}{|l|l|l|l|}
\hline Sample & $\begin{array}{l}\text { Enthalpy } \\
\text { (J/g) }\end{array}$ & $\begin{array}{l}\text { Crystallinity } \\
\text { of Mel (\%) }\end{array}$ & $\begin{array}{l}\text { Crystallinity of Mel } \\
\text { After 6 Months of } \\
\text { Storage (\%) }\end{array}$ \\
\hline $\begin{array}{l}\text { nanoMel } \\
\text { fluidMel }\end{array}$ & 12.24 & 13.43 & - \\
lyoMel & 36.54 & 12.98 & 13.02 \\
\hline
\end{tabular}

Abbreviation: Mel, meloxicam.

mins), followed by a slowing but rising profile. About $75 \%$ of Mel was dissolved within $2 \mathrm{hrs}$. In the case of the solid-state forms, carriers (MCC and trehalose) help to uniformly distribute the nanoparticles of Mel, thereby maintaining the uniqueness of the nanoparticles.

\section{In vivo Studies}

The plasma concentration of the samples in rats is shown in Figure 5. The calculated plasma concentration of Mel at $0 \mathrm{~min}$ $\left(\mathrm{C}_{0 \text { min }}\right)$ was $10,607 \mathrm{nM}$, and then the plasma concentration decreased exponentially. A very small amount of Mel was absorbed from the nanoMel sample, regardless of the particle size of the drug. The plasma concentration of Mel was constant in the investigated time period. The results show that the nanosuspension (nanoMel) has not got advantageous properties.

The initial blood levels of the fluidMel and lyoMel samples show a big difference. At 15 mins, the lyoMel sample $(5,712.98 \mathrm{nM})$ shows more than twice the value of fluidMel (Table 4). In practice, this value is similar to the

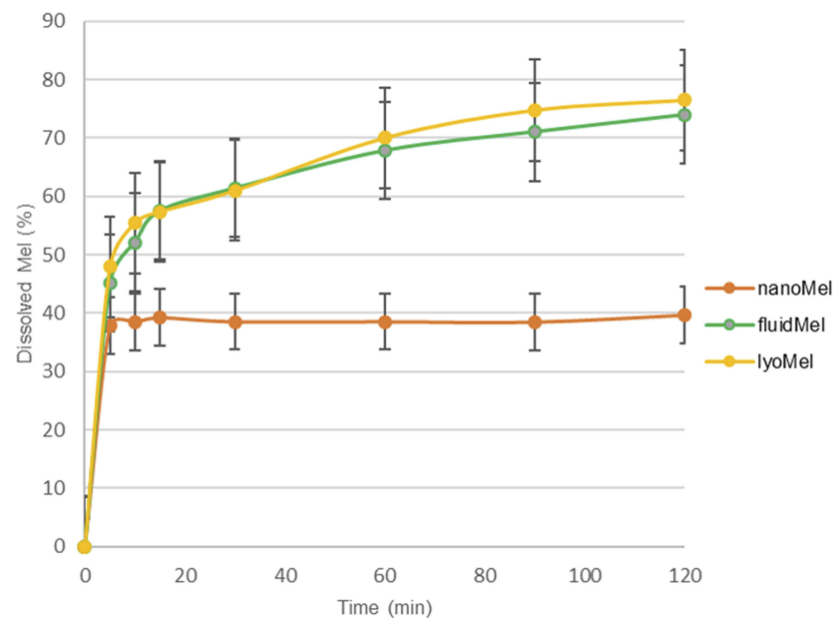

Figure $4 \mathrm{In}$ vitro dissolution of Mel from investigated samples. Medium: artificial gastric juice ( $\mathrm{pH}:$ I.2).

Abbreviation: Mel, meloxicam.

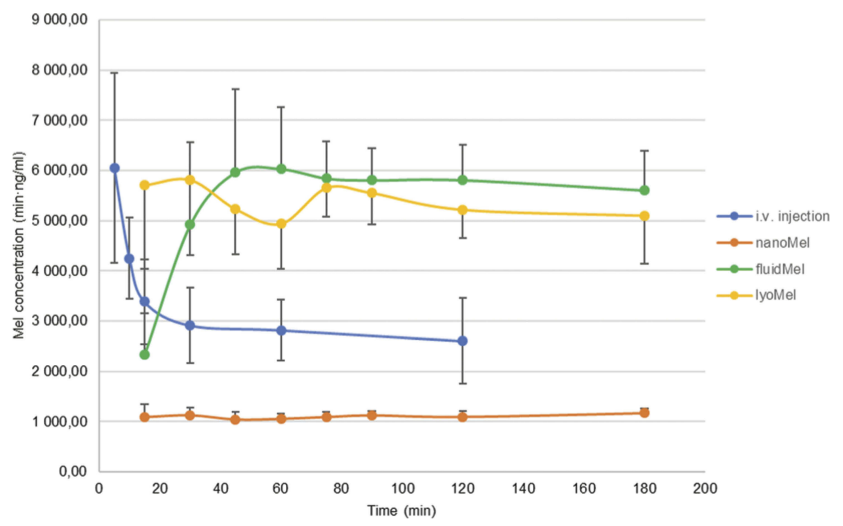

Figure 5 Plasma levels of MEL after the administration of different samples in rats. The preparations were administered orally (nanoMel, fluidMel and lyoMel) or intravenously (IV) as a single dose of $300 \mu \mathrm{g} / \mathrm{kg}$.

Abbreviation: Mel, meloxicam.

maximum plasma level for the lyophilized product $\left(\mathrm{C}_{30 \mathrm{~min}}\right.$ $5,814 \mathrm{nM}$ ). The peak blood concentration of fluidMel is about $6,000.00 \mathrm{nM}$ at 50 mins that is comparable with the blood concentration reached by IV injection at 5 mins. This result also confirms that the solid products contained Mel in an adequate amount and that the total amount thereof dissolved and absorbed.

The plasma curves of the different samples containing Mel show a very slow elimination after the distribution phases. That can be explained by the very high (99\%) plasma binding property of Mel in rat, and this ratio is the same in human. ${ }^{44}$ It seems that the eliminated portion of Mel is replenished from the protein bounded fraction for a quite long period of time. Our measuring time was only $3 \mathrm{hrs}$, longer detection period can provide appropriate information about the whole elimination process. The peak MEL concentrations of from lyoMel and fluidMel preparations have reached a similar level that of IV formula (Figure 5). The lyoMel sample resulted in higher plasma concentrations in 15 mins as compared with nanoMEL preparation. The solidified samples had nearly five-fold higher bioavailability than that of nanoMel (Table 4).

\section{IVIV Correlation}

Comparative studies according to AUC values have shown that there are significant differences between the nanosuspension and the samples (fluidMel and lyoMel) within in vitro and in vivo groups. However, there is no significant difference between two solid samples either in vitro or in vivo (Figure 6). The basis of the IVIVC calculation was the comparison of the AUC values of the samples in the in vitro and in vivo groups. By our calculations, the 
Table 4 Plasma Concentrations of Mel in Time and Its Relative Bioavailability in Rats After IV and per Os Administration of Mel Samples. Relative Bioavailabilities Were Compared to nanoMel Preparation

\begin{tabular}{|l|l|l|l|l|}
\hline Sample & $\mathbf{C}_{15 \min }(\mathbf{n M})$ & $\mathbf{C}_{\mathbf{1 2 0} \text { min }}(\mathbf{n M})$ & AUC $_{\text {blood }}(\mathbf{m i n} \mathbf{n g} / \mathbf{m L})$ & Relative Bioavailability (\%) \\
\hline nanoMel & $1,090.02 \pm 13.11$ & $1,123.31 \pm 14.24$ & $190,584.52$ & 100.00 \\
fluidMel & $2,338.44 \pm 17.25$ & $5,811.33 \pm 18.34$ & $945,834.99$ & 496.28 \\
lyoMel & $5,712.98 \pm 28.36$ & $5,219.52 \pm 20.86$ & $923,117.95$ & 484.36 \\
IV injection & $C_{5 \min } 6,059.07 \pm 15.76$ & $2,607.80 \pm 19.52$ & $377,528.01$ & - \\
\hline
\end{tabular}

Abbreviations: Mel, meloxicam; $\mathrm{C}_{15 \mathrm{~min}}, \mathrm{C}_{120 \mathrm{~min}}$, plasma concentrations of Mel in time; $\mathrm{AUC}_{\mathrm{blood}}$, area under the time-concentration curve $A U C$ interval is $\mathrm{t}_{0 \mathrm{~min}}-\mathrm{t}_{180 \text { min. }}$

Pearson's correlation coefficient value between the two studies is 0.99695 . The $\mathrm{t}$ value of the independent $t$-test of the two dissolution study series was 0.0145 , the calculated $\mathrm{p}$ value was 0.9889 and the difference is not significant at a confidence level of $95 \%$. As the zero hypothesis of the independent $t$-test, the calculation is not significant if the averages of the two series are equal. It can be concluded that in this system, in vitro dissolution studies are applicable to predict the dissolution rate-limited differences in the case of in vivo studies.

\section{Conclusion}

Mel-containing surfactant-free nanosuspension (nanoMel) as an intermediate product was produced by the wet milling process (planetary ball mill was combined with pearl milling technology). The energy invested in the milling decreased the mean particle size of Mel $(130 \mathrm{~nm})$ and broke the crystal structure of the nanoparticles (crystallinity index: $13.43 \%$ ). This can be considered a labile system, so stabilizing a suspension as a final dosage form without a surfactant does not work; therefore, the solidification of the nanoMel produced by fluidization and

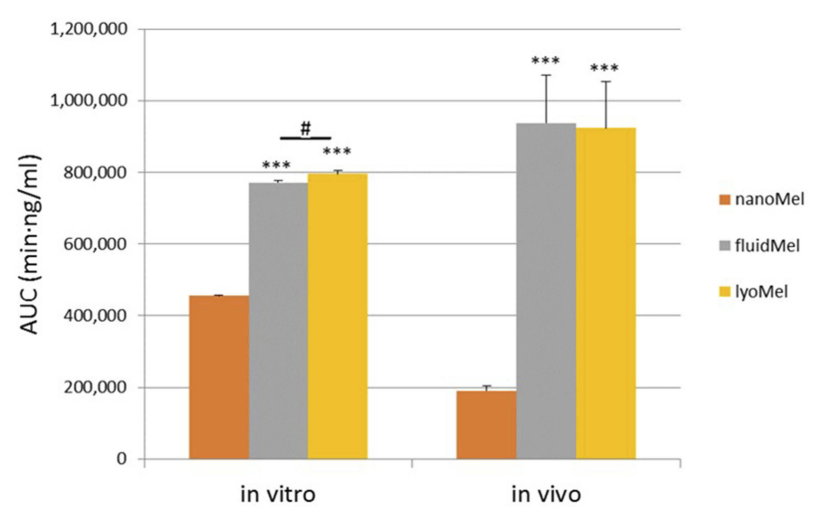

Figure 6 IVIV correlation of Mel-containing samples.

Notes: Values are presented as mean \pm SD. Statistically significant differences are: *** $\mathrm{p}<0.00 \mathrm{I}$, compared to nanoMel separately in in vitro and in vivo groups; \# $\mathrm{p}<0.05$ compared to the indicated columns.

Abbreviations: Mel, meloxicam; AUC, area under the time-concentration curve; IVIV correlation, in vitro-in vivo correlation; SD, standard deviation. lyophilization was chosen to ensure the critical product parameters (particle size, degree of crystallinity). We studied also the influence of solidification of nanoMel on the physical stability and drug bioavailability of the products. The nanoMel product had an optimized amount of PVA $(0.5 \%)$ as a protective polymer, but no surfactant as a further stabilizing agent because the final aim was to produce surfactant-free solid phase products as well.

The solidification studies showed that the critical product parameters of the intermediate product (nanoMel) were primarily provided by the fluidization technique which resulted in no significant change in mean particle size and crystallinity degree of Mel compared to the nanoMel. It is connected to the short operation time (50 mins), the large surface area of the MCC which fixed the nanoparticles with the sticking effect of the PVA and the crystallization inhibitory property of the fibers. ${ }^{33}$ The lyophilization required a longer operation time (72 hrs), which resulted in the amorphization of the crystalline carrier (trehalose $)^{45}$ and the recrystallization of Mel with an increased particle size and crystallinity degree. Finally, it was found that the physical stability of the solid phase products (fluidMel, lyoMel) was no change in particle size and crystallinity at 6 months of storage at room temperature $\left(23 \pm 2{ }^{\circ} \mathrm{C}, 45 \pm 5 \% \mathrm{RH}\right)$ compared to freshly measured products.

In our previous work (DDDT, 2018), ${ }^{10}$ there was performed a human Caco-2 intestinal epithelial cell line viability assay. Impedance measurement did not show significant cell damage after treatments with Mel, PVAMel formulations, as reflected by unchanged cell index values. The epithelial electrical resistance studies predicted the rapid penetration of nanonized Mel. In vivo studies justified the predicted data. The nanonized Mel in solidified products (fluidMel, lyoMel) resulted in rapid absorption through the gastric membrane by passive transcellular transport. It was found that the solid products contained $\mathrm{Mel}$ in an adequate amount and that the total amount thereof dissolved and absorbed. It has to be noted 
that Mel shows a very slow elimination because of very high plasma binding (99\%) in rat, and its replenishing time is quite a long period. MEL blood concentrations of lyoMel and fluidMel were similar to the level of IV form.

The solidified samples had nearly five-fold higher relative bioavailability than nanoMel application by oral administration and IVIV correlation was found between the in vitro and in vivo studies. The correlation between in vitro and in vivo studies showed that Mel nanoparticles fixed on the solid carrier (MCC, trehalose) in both the artificial gastric juice and the stomach of the animals rapidly reach saturation concentration leading to rapid absorption. These products show about 5 times greater bioavailability than the nanosuspension, in which the Mel nanoparticles can be aggregated in the stomach.

It can be stated that in the present study, the solidification of the nanosuspension (nanoMel) not only increased the stability of the nanoparticles but also allowed the preparation of surfactant-free solid compositions (powder, tablet, capsule), which may be an important consideration for certain groups of patients to achieve rapid analgesia. Further experiments are necessary to prove the therapeutic relevance of these innovative formulations.

\section{Acknowledgments}

This work was supported by the National Research Development and Innovation Office and Richter Plc., Budapest, Hungary (GINOP 2.2.1 15-2016-00007). The grant (20391-3/2018/FEKUSTRAT) of the Ministry of Human Capacities, Hungary is acknowledged.

\section{Author Contributions}

All authors contributed to data analysis, drafting or revising the article, gave final approval of the version to be published, and agree to be accountable for all aspects of the work.

\section{Disclosure}

The authors report no financial interest and no conflicts of interest in this work.

\section{References}

1. Müller RH, Benita S, Böhm BHL. Emulsions and Nanosuspensions for the Formulation of Poorly Soluble Drugs. Stuttgart, Germany: Medpharm Scientific Publishers. 1998;149-174.

2. Dahiya S, Pathak K, Dahiya R, et al. Nanoparticle engineering processes: industrial technologies for delivery of poorly water-soluble drugs. Egypt Pharm J. 2007;6:87-101.
3. Meng L, Mohammad A, Rajesh D, et al. Nanomilling of drugs for bioavailability enhancement: a holistic formulation-process perspective. Pharmaceutics. 2016;8:17-52. doi:10.3390/pharmaceutics8020017

4. Sharma P, denny WA, Garg S. Effect of wet milling process on the solid state of indomethacin and simvastatin. Int $J$ Pharm. 2009;380:40-48. doi:10.1016/j.ijpharm.2009.06.029

5. Malamatari M, Somavarapu S, Taylor KMG, et al. Solidification of nanosuspensions for the production of solid oral dosage forms and inhalable dry powders. Exp Opin Drug Del. 2016;13:435-450. doi:10.1517/17425247.2016.1142524

6. Lee J, Cheng Y. Critical freezing rate in freeze drying nanocrystal dispersions. J Control Release. 2006;111:185-192. doi:10.1016/j. jconrel.2005.12.003

7. Liversidge GG, Cundy KC. Particle size reduction for improvement of oral bioavailability of hydrophobic drugs. I. Absolute oral bioavailability of nanocrystalline danazol in beagle dogs. Int $J$ Pharm. 1995;125:91-97. doi:10.1016/0378-5173(95)00122-Y

8. Ding Z, Wang L, Xing Y, Zhao Y, Wang Z, Han J. Enhanced oral bioavailability of celecoxib nanocrystalline solid dispersion based on wet media milling technique: formulation, optimization and in vitro/ in vivo evaluation. Pharmaceutics. 2019;11:328-346. doi:10.3390/ pharmaceutics 11070328

9. Wiedmann TS, DeCastro L, Wood RW. Nebulization of nanoCrystalsTM: production of a respirable solid-in-liquid-in-air colloidal dispersion. Pharm Res. 1997;14:112-116. doi:10.1023/A:1012024021511

10. Bartos C, Jójárt-Laczkovich $\mathrm{O}$, Katona $\mathrm{G}$, et al. Optimization of a combined wet milling process in order to produce poly(vinyl alcohol) stabilized nanosuspension. Drug Des Dev Ther. 2018;12:1567-1580. doi:10.2147/DDDT.S159965

11. Chiang PC, Wahlstrom JL, Selbo JG, et al. 1,3- Dicyclohexyl urea nanosuspension for intravenous steady-state delivery in rats. $J$ Exp Nanosci. 2007;2:239-250. doi:10.1080/17458080601134540

12. Zhang Y, Fei S, Yu M, et al. Injectable sustained release PLA microparticles prepared by solvent evaporation-media milling technology. Drug Dev Ind Pharm. 2018;44:1591-1597. doi:10.1080/036390 45.2018.1483382

13. Girdhar V, Patil S, Banerjee S, Singhvi G. Nanocarriers for drug delivery: mini review. Recent Pat Nanomed. 2018;8:88-99.

14. Ostrander KD, Bosch HW, Bondanza DM. An in-vitro assessment of a nanoCrystalTM beclomethasone dipropionate colloidal dispersion via ultrasonic nebulization. Eur J Pharm Biopharm. 1999;48:207-215. doi:10.1016/S0939-6411(99)00049-1

15. Vergote GJ, Nervaet C, Van Driessche I, et al. An oral controlled release matrix pellet formulation containing nanocrystalline ketoprofen. Int J Pharm. 2001;2019:81-87.

16. Merisko-Liversidge E, Sarpotdar P, Bruno J, et al. Formulation and antitumor activity evaluation of nanocrystalline suspensions of poorly soluble anticancer drugs. Pharm Res. 1996;13:272-278. doi:10.1023/ A:1016051316815

17. Siewert C, Moog R, Alex R, Kretzer P, Rothenhäusler B. Process and scaling parameters for wet media milling in early phase drug development: a knowledge-based approach. Eur $J$ Pharm Sci. 2018;115:126-131. doi:10.1016/j.ejps.2017.12.022

18. Peltonen L, Hirvonen J. Pharmaceutical nanocrystals by nanomilling: critical process parameters, particle fracturing and stabilization methods. J Pharm Pharmacol. 2010;62:1569-1579. doi:10.1111/ j.2042-7158.2010.01022.x

19. Kim CJ. Surface Chemistry and Colloids in Advanced Pharmaceutics: Physico-Chemical Principles. Florida, USA: CRC Press; 2004:193-256.

20. Derjaguin BV, Landau L. Theory of the stability of strongly charged lyophobic sols and of the adhesion of strongly charged particles in solutions of electrolytes. Acta Phys Chem. 1941;14:633-662.

21. Verma S, Kumar S, Gokhale R, Burgess JD. Physical stability of nanosuspensions: investigation of the role of stabilizers on Ostwald ripening. Int J Pharm. 2011;406:145-152. doi:10.1016/j.jpharm.2010.12.027 
22. Bhakay A, Azad M, Vizzotti E, et al. Enhanced recovery and dissolution of griseofulvin nanoparticles from surfactant-free nanocomposite microparticles incorporating wet-milled swellable dispersants. Drug Dev Ind Pharm. 2014;40:1509-1522. doi:10.3109/03639045.2013.831442

23. Azad M, Afolabi A, Bhakay A, et al. Enhanced physical stabilization of fenofibrate nanosuspensions via wet co-milling with a super disintegrant and an adsorbing polymer. Eur J Pharm Biopharm. 2015;94:372-385. doi:10.1016/j.ejpb.2015.05.028

24. Hye_in K, Sang YP, Seok JP, et al. Development and evaluation of a reconstitutable dry suspension ti improve the dissolution and oral absorption of poorly water-soluble celecoxib. Pharmceutics. 2018;10:140. doi:10.3390/pharmaceutics10030140

25. Wu L, Zhang J, Watanabe W. Physical and chemical stability of drug nanoparticles. Adv Drug Deliv Rev. 2011;63:456-469. doi:10.1016/j. addr.2011.02.001

26. Wang Y, Zheng Y, Zhang L, et al. Stability of nanosuspensions in drug delivery. J Control Release. 2013;172:1126-1141. doi:10.1016/ j.jconrel.2013.08.006

27. Van Eerdenbrugh B, Froyen L, Van Humbeeck J, et al. Drying of crystalline drug nanosuspensions - the importance of surface hydrophobicity on dissolution behavior upon redispersion. Eur J Pharm Sci. 2008;35:127-135. doi:10.1016/j.ejps.2008.06.009

28. Kumari Prasad L, Smyth H. 3D Printing technologies for drug delivery: a review. Drug Dev Ind Pharm. 2016;42:1019-1031. doi:10.3109/03639045.2015.1120743

29. Colombo M, Orthmann S, Bellini M, et al. Influence of drug brittleness, nanomilling time, and freeze-drying on the crystallinity of poorly water-soluble drugs and its implications for solubility enhancement. AAPS Pharm Sci Tech. 2017;18:2437-2445. doi:10.1208/s12249-017-0722-4

30. Kayaert P, Anné M, Van den Mooter G. Bead layering as a process to stabilize nanosuspensions: influence of drug hydrophobicity on nanocrystal reagglomeration following in-vitro release from sugar beads. J Pharm Pharmacol. 2011;63:1446-1453. doi:10.1111/j.2042-7158.2011.01351.x

31. He W, Lu Y, Qi J, et al. Formulating food protein-stabilized indomethacin nanosuspensions into pellets by fluid-bed coating technology: physical characterization, redispersibility, and dissolution. Int J Nanomedicine. 2013;8:3119-3128. doi:10.2147/IJN.S46207
32. Möschwitzer J, Müller RH. Spray coated pellets as carrier system for mucoadhesive drug nanocrystals. Eur $J$ Pharm Biopharm. 2006;62:282-287. doi:10.1016/j.ejpb.2005.09.005

33. Jójárt-Laczkovich O, Szabó-Révész P. Formulation of tablets containing an 'in-process' amorphized active pharmaceutical ingredient. Drug Dev Ind Pharm. 2011;37:1272-1281. doi:10.3109/03639045.2011.569933

34. Szabó-Révész P. Modifying the physicochemical properties of NSAIDs for nasal and pulmonary administration. Drug Discov Today Technol. 2018;27:87-93. doi:10.1016/j.ddtec.2018.03.002

35. Simmons DL, Botting RM, Hla T. Cyclooxygenase isozymes: the biology of prostaglandin synthesis and inhibition. Pharmacol Rev. 2004;56:387-437. doi:10.1124/pr.56.3.3

36. Bartos C, Szabó-Révész P, Bartos C, et al. The effect of an optimized wet milling technology on the crystallinity, morphology and dissolution properties of micro- and nanonized meloxicam. Molecules. 2016;21:507-518. doi:10.3390/molecules21040507

37. Abramoff MD, Magelhaes PJ, Ram SJ. Image processing with image. J Biophotonics Int. 2004;11:36-42.

38. Mártha C, Kürti L, Farkas G, et al. Effects of polymers on the crystallinity of nanonized meloxicam during a co-grinding process. Eur Polym J. 2013;49:2426-2432. doi:10.1016/j.eurpolymj.2013.03.006

39. Aulton ME, Livingstone C. Aulton's Pharmaceutics, the Design and Manufacture of Medicines. Elsevier; 2007.

40. Cardot JM, Davit BM. In vitro-in vivo correlations: tricks and traps. AAPS J. 2012;14:491-499. doi:10.1208/s12248-012-9359-0

41. Kevin J. Using nondestructive in-situ measurements to ensure lyophilized product stability. Biopharm Int. 2018;31:24-25.

42. Kian LK, Jawaid M, Ariffin H, et al. Isolation and characterization of microcrystalline cellulose from roselle fibers. Int Biol Macromol. 2017;103:931-940. doi:10.1016/j.ijbiomac.2017.05.135

43. Sussich $\mathrm{F}$, Cesàro $\mathrm{A}$. Trehalose amorphization and recrystallization. Carbohyd Rev. 2008;343:2667-2674. doi:10.1016/j.carres.2008.08.008

44. Busch U, Schmid J, Heinzel G, et al. Pharmacokinetics of meloxicam in animals and the relevance to humans. Drug Metab Dispos. 1998;26:576-584

45. Jójárt-Laczkovich O, Katona G, Aigner Z, et al. Investigation of recrystallization of amorphous trehalose through hot-humidity stage X-ray powder diffraction. Eur J Pharm Sci. 2016;95:145-151. doi:10.1016/j.ejps.2016.08.003

\section{Publish your work in this journal}

Drug Design, Development and Therapy is an international, peerreviewed open-access journal that spans the spectrum of drug design and development through to clinical applications. Clinical outcomes, patient safety, and programs for the development and effective, safe, and sustained use of medicines are a feature of the journal, which has also been accepted for indexing on PubMed Central. The manuscript management system is completely online and includes a very quick and fair peer-review system, which is all easy to use. Visit http://www. dovepress.com/testimonials.php to read real quotes from published authors. 